\title{
Risk of Developing Posttraumatic Stress Disorder in Severe COVID- 19 Survivors, their Families and Frontline Healthcare Workers: What Should Mental Health Specialists Prepare For?
}

\author{
Marcin Sekowski $^{1 *}$, Malgorzata Gambin ${ }^{2}$, Karolina Hansen ${ }^{2}$, Pawel Holas ${ }^{2}$, Sylwia \\ Hyniewska $^{3}$, Julia Wyszomirska ${ }^{4}$, Agnieszka Pluta ${ }^{2}$, Marta Sobańska ${ }^{2}$, Emilia Lojek ${ }^{2}$ \\ ${ }^{1}$ Department of Psychology, The Maria Grzegorzewska University, Warsaw, Poland \\ ${ }^{2}$ Department of Psychology, University of Warsaw, Warsaw, Poland \\ ${ }^{3}$ Department of Experimental Psychology, University College London, United Kingdom \\ ${ }^{4}$ Department of Psychology, Medical University of Silesia in Katowice, Poland \\ * Correspondence:
}

Marcin Sekowski, Department of Psychology, The Maria Grzegorzewska University, ul. Szczesliwicka 40, 02-353 Warszawa, Poland, msekowski@aps.edu.pl

This manuscript has been accepted for publication in the Frontiers in Psychiatry.

Sekowski, M., Gambin, M., Hansen, K., Holas, P., Hyniewska, S., Wyszomirska, J., Pluta, A., Sobańska, M., \& Łojek'E. (2021). Risk of developing posttraumatic stress disorder in severe COVID-19 survivors, their families and frontline healthcare workers: What should mental health specialists prepare for? Frontiers in Psychiatry.

https://doi.org/10.3389/fpsyt.2021.562899

\begin{abstract}
Given the high coronavirus disease 2019 (COVID-19) mortality, this disease may be a lifethreatening event, especially for individuals at high risk of complications. Therefore, in the article we try to answer two questions that are relevant to public mental health: Can we define groups who are at higher risk of developing pandemic-related PTSD? How can health specialists prepare for it? Given the results of previous research on PTSD in epidemic (e.g. SARS) survivors, we suggest that mental health professionals in countries touched by the pandemic should prepare for an increase in the PTSD prevalence, specifically in: individuals who have had severe COVID-19; family members of these patients and of patients who have died; and frontline healthcare workers witnessing COVID-19 patients' sudden deaths, or numerous life-threatening situations. We postulate that these groups at risk should be routinely screened for PTSD in primary medical and pediatric care. Mental health services should prepare for providing therapeutic interventions for individuals with PTSD in the vulnerable groups, and support to their families, especially children.
\end{abstract}

Keywords: coronavirus disease 2019 (COVID-19), posttraumatic stress disorder (PTSD), severe acute respiratory syndrome (SARS), prolonged grief disorder (PGD) 


\section{Introduction}

The COVID-19 pandemic covered 223 countries, 136,291,755 individuals with confirmed infection and 2,941,128 deaths as of April 13, 2021 (WHO, 2021). Globally the case mortality rate from COVID-19 is 10.4 deaths per 100,000 inhabitants but it varies widely by region and country, with as high as 84.9 in Belgium, 67.5 in Andorra, and 64.3 in the United Kingdom (Hashim et al., 2020). Age is the main risk factor for complications and death as a result of COVID-19; the disease death rate varies from $0.2 \%$ for individuals aged 10 to 39 years to $21.9 \%$ in people over 80 years. Death rate also increases in patients with preexisting comorbid conditions, such as history of metastatic solid tumor (57\%), myocardial infarction (47\%), cerebrovascular disease (39\%), congestive heart failure (37\%), hemiplegia (34\%), malignant neoplasm (27\%), diabetes (20\%), dementia (20\%), chronic pulmonary disease (16\%), hyperlipidemia (11\%), and hypertension (8.4\%; Rosenthal, et al. 2020; The Novel Coronavirus Pneumonia Emergency Response Epidemiology Team, 2020; WHO, 2020c). Given the high COVID-19 mortality, the severe disease may be life-threatening and as such may be considered a traumatic event, especially for individuals at high risk of complications and death (Bo et al., 2020; Janiri et al., 2021; Tarsitani et al., 2021).

People who are exposed to traumatic events can develop posttraumatic stress disorder (PTSD). PTSD is a serious mental disorder that can develop in persons exposed not only to actual or threatened death who directly experienced the traumatic event(s), but also in those who witnessed such an event personally, learned that the accidental or violent event occurred to a close family member, or friend or experienced repeated or extreme exposure to aversive details of the traumatic event personally. Such an event is criterion A for PTSD in the Diagnostic and Statistical Manual of Mental Disorders, Fifth Edition (DSM-5; American Psychiatric Association [APA], 2013). PTSD causes severe distress or impairment in psychosocial functioning and is marked by four main types of symptoms that last for at least a month, being respectively the criteria B, C, D and E for the disorder: (i) intrusions associated with the traumatic event, e.g. involuntary distressing event-related memories, dreams, and flashbacks, or physiological reactions to cues that resemble the traumatic event; (ii) avoidance of event-related stimuli, i.e. internal (memories, thoughts and feelings) or external (people, places, situations) reminders; (iii) negative alterations in event-related cognitions, and/or mood, e.g. inability to remember an important aspect of the event, distorted cognitions about the event leading to blaming himself or herself or others and/or persistent negative emotional 
state and inability to experience positive emotions, diminished activities, and/or detachment from others; and (iv) event-related increased arousal and reactivity ,e.g. difficulty sleeping and concentrating, reckless or self-destructive behavior, and being easily irritated and angered (APA, 2013). PTSD individuals are 2-5 times more at-risk of suicidal ideation, suicide attempt, and deaths by suicide (Thibodeau et al., 2013). Previous research indicates that about $80 \%$ of individuals with PTSD have at least one other comorbid disorder, in particular: depressive and anxiety disorders, as well as substance use disorder (e.g. Galatzer-Levy et al., 2013; Rytwinski, et al., 2013).

Given the exceptional epidemic situation we are facing now, can we define groups at high risk of developing pandemic-related PTSD? What steps can mental health specialists take to prepare for it? In the following paper we will briefly address these issues.

\section{What Groups Have High Risk of Developing Pandemic-related PTSD?}

We believe that mental health professionals in pandemic countries should be prepared for an increase in the PTSD prevalence in three specific groups during COVID-19 pandemic and later: (i) individuals who have had severe COVID-19 and who feared imminent death from the disease; (ii) family members of these severely touched patients or patients who have died as a result of the disease; and (iii) frontline healthcare workers (HCWs) witnessing COVID-19 patients' sudden deaths, or life-threatening situations.

\subsection{Severe COVID-19 Survivors}

Among COVID-19 individuals, those with a severe course of the disease are particularly at risk of developing PTSD (Bo et al., 2020; Janiri et al., 2021; Tarsitani et al., 2021). Patients who had to undergo medical interventions to maintain or restore vital functions experience particularly intense traumatic stress (Chambarlain et al., 2021; Girard et al., 2007). A very traumatically stressed subgroup may also include individuals with severe disease who were refused health care as a result of health service failure during a pandemic (Huang et al., 2016; Lee et al., 2007). The meta-analysis of 35 studies involving 79,170 people with COVID-19 (Li et al., 2021) showed that almost a quarter of them (23\%) develop a severe form of the disease (35 studies, 79,170 patients) which requires close monitoring. The total percentage of patients admitted to the intensive care unit was $11.0 \%$ (39 studies, 80,487 patients), with no significant differences between various countries or regions (Li et al. 2021). The life of many of these individuals is threatened and many of them may experience high levels of fear of imminent death, which creates a traumatic event. 
The effects of the current as well as of past epidemics on the mental health of survivors can help predict the psychopathological consequences of a severe life-threatening viral disease. Two Italian studies on recovered severe COVID-19 survivors reported that the prevalence of PTSD is 10.4\% (Tarsitani et al., 20121) and 30.2\% (Janiri et al., 2021), respectively. Additionally, in survivors of the severe acute respiratory syndrome (SARS) during the epidemic that covered 29 countries in 2002/2003, infecting 8,096 individuals, of whom 774 died PTSD was present in $39 \%$ of patients 10 months after discharge (Hong et al., 2009), and over time the frequency of PTSD even increased, ranging from $42 \%$ to $54.5 \%$ after 31-51 months from discharge (Hong et al., 2009; Lam et al., 2009; Mak et al., 2009). Given that an estimated $3.6 \%$ of U.S. adults had PTSD in the former year, and the lifetime prevalence of this syndrome being $6.8 \%$ (Koenen et al., 2017), the cited studies indicate that PTSD is a common chronic mental health problem following a virus disease with high mortality (Gardner \& Moallef, 2015).

It should be added that COVID-19 infected persons most often develop less severe symptoms such as fever (78.8\%), loss of smell and/or taste (from $19.0 \%$ to $73.6 \%$ ), cough (53.9\%) and malaise (37.9\%), and sometimes diarrhea (9.5\%), rhinitis (7.5\%), and abdominal pain and vomiting (4.5\%) (Aggarwal et al., 2020; Li et al., 2021; Vaira et al., 2020) which are usually not life-threatening and are treated at home with over-the-counter medications. Infection may also be asymptomatic (Tong et al., 2020; Wei et al., 2020; Arons et al., 2020). Such cases are not likely to develop PTSD as COVID-19 infection in most of the cases does not lead to symptoms severe enough to be life threatening and these individuals do not meet the A criterion for PTSD (APA, 2013). However, some persons, although not hospitalized, may experience severe symptoms of COVID-19 and fear of imminent death, thereby experiencing a traumatic event, and can develop PTSD; high levels of fear of death during the event, not the type of medical treatment, are the hallmark of a traumatic stressor.

\subsection{Families of Severe COVID-19 Survivors and of Persons Who Died of the Disease}

The second group at risk of developing pandemic-related PTSD are the families of people whose lives were at risk due to severe COVID-19 as well as the families of those who died of the disease under emergency circumstances. Being a witness to the threat to the life of another person, especially a loved one in the course of COVID-19, can be a traumatic event (APA, 2013). The family's situation becomes even more complicated when a loved one has died in the course of the disease. Bereaved persons can then be obliged not only to deal with the traumatic events of life-threatening viral disease and the death of a loved one, but also 
with intense grief symptoms. The sudden death of a loved one and the inability to prepare for the death - which often occurs during a pandemic - is a risk factor for prolonged grief disorder (Sekowski \& Prigerson, 2021). Grief of family members may be additionally difficult and complicated due to the circumstances surrounding death and mourning during a pandemic, e.g. quarantine of the dying persons, and delayed and very modest funerals. Grief and mourning in this time, due to accumulation of death and difficulties with recognizing individual bereavement and pain during pandemic, especially after loss of an elderly with chronic diseases, can be disenfranchising and therefore additionally complicated (KokouKpolou et al., 2020).

\subsection{Frontline Healthcare Workers}

The third group at risk of developing PTSD during a pandemic are healthcare professionals who experience traumatic stress in the context of witnessing patients' dying and their lives being threatened, especially in cases of insufficient or inadequate intensive medical equipment. Furthermore, shortages of protective or medical equipment (Chen et al., 2020; Greenberg et al., 2020) can be potentially traumatic if they lead the HCWs to fear of contamination and own imminent death. Own severe course of the disease and being a witness to the threat to life or death of other people may lead to the accumulation of traumatic stress in HCWs. Studies showed that after the SARS epidemic in 2002/2003, PTSD developed more often in infected HCWs than in non-HCWs (Mak et al., 2009, 2010). We may predict that other professional groups such as employees of nursing homes and of other long-term care facilities also may experience some of these above mentioned traumatic stress factors (McMichael et al., 2020).

\section{$3 \quad$ What Steps Can Be Taken to Prepare for Pandemic-related PTSD?}

A systematic approach to services to prevent, diagnose, and treat PTSD in risk groups is needed (Boden et al., 2021). First of all, mental health experts should be aware of the risk of developing PTSD in severe COVID-19 survivors who feared imminent death from the disease, their family members and family members of patients who have died as a result of the pandemic, as well as frontline HCWs witnessing patients' sudden deaths, or life-threatening situations. Information about PTSD and its symptoms should be made available to all HCWs and to mental health patients and their families during and in the months following the pandemic. 
Second, we postulate that the three groups at risk described above should be routinely screened for PTSD in the months following the onset of the disease as the usual window of PTSD onset lies between 1 and 6 months after a traumatic event. Screening should be done in primary medical and pediatric care if resources are immediately available for timely followup by mental health professionals with specific expertise in PTSD assessment, and - in optimal circumstances - treatment of this disorder. Screening should distinguish between other mental health problems versus PTSD. Measurement should begin with screening first for traumatic events, i.e. directly experiencing or witnessing death or imminent threat of death due to the pandemic (or other concurrent or previous incidents) being criterion $A$ of the disorder according to DSM-5. PTSD symptoms should be screened only if potentially traumatic events are identified. For example, non-symptomatic individuals who test positive for COVID-19 are not at risk and should not be screened for PTSD, although they may develop other mental disorders such as depressive or anxiety disorder (Mazza et al., 2020). Co-occurrence of PTSD and other disorders (see Janiri et al., 2021; Tarsitani et al., 2021) is associated with high levels of distress, burden and reduced quality of life (e.g. Galatzer-Levy et al., 2013; Rytwinski et al., 2013), thus special clinical attention should be paid to individuals with PTSD experiencing additional mental health problems.

Bereaved surviving close ones who died unexpectedly as a result of the pandemic should be screened not only for PTSD but also for prolonged grief disorder, for example with the Prolonged Grief Disorder-13-Revised scale (Prigerson et al., 2021). PGD measurement should be carried out 12 months after the loss, as - according to DSM-5-TR - this is the time after which the persistence of severe grief symptoms, if they interfere with the psychosocial functioning, indicates the presence of this disorder (APA, 2020).

Third, mental health services should prepare to provide evidence-based therapeutic interventions for individuals with PTSD such as prolonged exposure therapy, and cognitive processing therapy (Cusack et al., 2016). It would be worth training clinicians in various countries in delivering these evidence-based interventions online, as well as to test their effectiveness in individuals with pandemic-related PTSD. Moreover, mental health professionals should be encouraged to: (i) share their experiences on the adjustments to be made in order to tailor these interventions to the current situation, e.g. necessity to provide interventions online and to acknowledge the specificity of the clinical picture of symptoms in severe COVID-19 survivors and their families (including the bereaved), and HCWs; (ii) define frameworks to effectively implement evidence-based interventions in mental health 
services in various countries (in particular in low income countries); and (iii) prepare accessible and easy-to-follow online trainings for professionals from various countries on evidence-based therapeutic interventions for PTSD.

Finally, studies indicate that the severity of PTSD symptoms in parents is associated with an increase in behavioral and emotional problems in their children (e.g. Parsons et al., 2018; Kritikos et al., 2019). Therefore, one should be prepared to prevent such cascading effects of the pandemic by considering psychological support for children of parents who have developed PTSD due to the pandemic. Again, one of the challenges would be providing support for children and their families through online services.

The issue of the full prevalence of pandemic-related PTSD remains a matter of future research as symptoms may develop up to 6 months after the traumatic event. Research showing the full scope of pandemic-related PTSD prevalence will be possible only a few months after the end of the outbreak. Former studies indicated a large prevalence of PTSD in survivors of the earlier coronavirus epidemic (SARS), especially in HCWs, therefore we suggest that mental health professionals should prepare for the frequent occurrence of PTSD in this high risk groups. Families of survivors of severe diseases with high mortality and those who died from the pandemic, as well as frontline HCWs who were not infected but have witnessed numerous deaths and life-threatening situations - although not included in such systematic studies as survivors - may also experience traumatic stress during the outbreak and develop PTSD. Notwithstanding, we suggest that health care policies should consider routine screening for the presence of PTSD symptoms in the three groups at risk described in this paper, together with preventive and treatment strategies of PTSD, and related risks such as suicide.

\section{Conflict of Interest}

The authors declare that the research was conducted in the absence of any commercial or financial relationships that could be construed as a potential conflict of interest.

\section{$5 \quad$ Author Contributions}

Marcin Sekowski wrote the first draft of the manuscript. All authors reviewed, edited, and wrote the manuscript.

\section{Acknowledgments}


The very first form of the manuscript have previously appeared online as a preprint: https://psyarxiv.com/bnkve/

\section{$7 \quad$ References}

Aggarwal, S., Garcia-Telles, N., Aggarwal, G., Lavie, C., Lippi, G., \& Henry, B. M. (2020). Clinical features, laboratory characteristics, and outcomes of patients hospitalized with coronavirus disease 2019 (COVID-19): Early report from the United States. Diagnosis (Berlin, Germany), 7(2), 91-96. https://doi.org/10.1515/dx-2020-0046

American Psychiatric Association. (2013). Diagnostic and Statistical Manual of Mental Disorders (5th Ed.). American Psychiatric Association: Washington, DC. https://doi.org/10.1176/appi.books.9780890425596

American Psychiatric Association. (2020). View and comment on recently proposed changes to DSM-5. Addition of a new diagnosis, "Prolonged Grief Disorder". https://www.psychiatry.org/psychiatrists/practice/dsm/proposed-changes

Bo, H. X., Li, W., Yang, Y., Wang, Y., Zhang, Q., Cheung, T., Wu, X., \& Xiang, Y. T. (2020). Posttraumatic stress symptoms and attitude toward crisis mental health services among clinically stable patients with COVID-19 in China. Psychological Medicine, 27, 1-7. https://doi.org/10.1017/S0033291720000999

Boden, M., Zimmerman, L., Azevedo, K. J., Ruzek, J. I., Gala, S., Abdel Magid, H. S., Cohen, N., Walser, R., Mahtani, N. D., Hoggatt, K. J., \& McLean, C. P. (2021). Addressing the mental health impact of COVID-19 through population health. Clinical Psychology Review, 85, 102006. https://doi.org/10.1016/j.cpr.2021.102006

Chamberlain, S. R., Grant, J. E., Trender, W., Hellyer, P., \& Hampshire, A. (2021). Posttraumatic stress disorder symptoms in COVID-19 survivors: online population survey. BJPsych open, 7(2). https://doi.org/10.1192/bjo.2021.3

Chen, Q., Liang, M., Li, Y., Guo, J., Fei, D., Wang, L., He, L., Sheng, C., Cai, Y., Li, X., Wang, J., \& Zhang, Z. (2020). Mental health care for medical staff in China during the COVID-19 outbreak. The Lancet Psychiatry, 7(4), e15-e16. https://doi.org/10.1016/S2215-0366(20)30078-X

Cusack, K., Jonas, D. E., Forneris, C. A., Wines, C., Sonis, J., Middleton, J. C., Feltner, C., Brownley, K. A., Olmsted, K. R., Greenblatt, A., Weil, A., \& Gaynes, B. N. (2016). Psychological treatments for adults with posttraumatic stress disorder: A systematic 
review and meta-analysis. Clinical Psychology Review, 43, 128-141.

https://doi.org/10.1016/j.cpr.2015.10.003

Galatzer-Levy, I. R., Nickerson, A., Litz, B. T., \& Marmar, C. R. (2013). Patterns of lifetime PTSD comorbidity: A latent class analysis. Depression and Anxiety, 30(5), 489-496. https://doi.org/10.1002/da.22048

Gardner, P. J., \& Moallef, P. (2015). Psychological impact on SARS survivors: Critical review of the English language literature. Canadian Psychology/Psychologie Canadienne, 56(1), 123-135. https://doi.org/10.1037/a0037973

Girard, T. D., Shintani, A. K., Jackson, J. C., Gordon, S. M., Pun, B. T., Henderson, M. S., ... \& Ely, E. (2007). Risk factors for post-traumatic stress disorder symptoms following critical illness requiring mechanical ventilation: a prospective cohort study. Critical care, 11(1), 1-8. https://doi.org/10.1186/cc5708

Greenberg, N., Docherty, M., Gnanapragasam, S., \& Wessely, S. (2020). Managing mental health challenges faced by healthcare workers during COVID-19 pandemic. BMJ, 368. https://doi.org/10.1136/bmj.m1211Hashim, M. J., Alsuwaidi, A. R., \& Khan, G. (2020). Population risk factors for COVID-19 mortality in 93 countries. Journal of Epidemiology and Global Health, 10(3), 204-208.

https://doi.org/10.2991/jegh.k.200721.001

Hong, X., Currier, G. W., Zhao, X., Jiang, Y., Zhou, W., \& Wei, J. (2009). Posttraumatic stress disorder in convalescent severe acute respiratory syndrome patients: A 4-year followup study. General Hospital Psychiatry, 31, 546-554. https://doi.org/10.1016/j.genhosppsych.2009.06.008

Huang, M., Parker, A. M., Bienvenu, O. J., Dinglas, V. D., Colantuoni, E., Hopkins, R. O., Needham, D. M., \& National Institutes of Health, National Heart, Lung, and Blood Institute Acute Respiratory Distress Syndrome Network (2016). Psychiatric symptoms in acute respiratory distress syndrome survivors: A 1-year national multicenter study. Critical Care Medicine, 44, 954-965. https://doi.org/10.1097/CCM.0000000000001621

Janiri, D., Carfi, A., Kotzalidis, G. D., Bernabei, R., Landi, F., Sani, G., Gemelli Against COVID-19 Post-Acute Care Study Group. (2021) Posttraumatic stress disorder in patients after severe COVID-19 infection. JAMA Psychiatry. Advance online publication. https://doi.org/10.1001/jamapsychiatry.2021.0109 
Koenen, K. C., Ratanatharathorn, A., Ng, L., McLaughlin, K. A., Bromet, E. J., Stein, D. J., Karam, E. G., Ruscio, A. M., Benjet, C., Scott, K., Atwoli, L., Petukhova, M., Lim, C. C. W., Aguilar-Gaxiola, S., Al-Hamzawi, A., Alonso, J., Bunting, B., Ciutan, M., de Girolamo, G., . . WHO World Mental Health Survey collaborators. (2017). Posttraumatic stress disorder in the World Mental Health Surveys. Psychological Medicine, 47(13), 2260-2274. https://doi.org/10.1017/S0033291717000708

Kokou-Kpolou, C. K., Fernandez-Alcantara, M., \& Cenat, J. M. (2020). Prolonged grief related to COVID-19 deaths: Do we have to fear a steep rise in traumatic and disenfranchised griefs? Psychological Trauma, 12(S1), S94-S95. https://doi.org/10.1037/tra0000798

Kritikos, T. K., Comer, J. S., He, M., Curren, L. C., \& Tompson, M. C. (2019). Combat experience and posttraumatic stress symptoms among military-serving parents: A meta-analytic examination of associated offspring and family outcomes. Journal of Abnormal Child Psychology, 47(1), 131-148. https://doi.org/10.1007/s10802-0180427-5

Lam, M. H.-B., Wing, Y.-K., Yu, M. W.-M., Leung, C.-M., Ma, R. C. W., Kong, A. P. S., So, W. Y., Fong, S. Y.-Y., \& Lam, S.-P. (2009). Mental morbidities and chronic fatigue in severe acute respiratory syndrome survivors. Archives of Internal Medicine, 169, 2142-2147. https://doi.org/10.1001/archinternmed.2009.384

Lee, A. M., Wong, J. G. W. S., McAlonan, G. M., Cheung, V., Cheung, C., Sham, P. C., Chu, C.-M., Wong, P.-C., Tsang, K. W. T., \& Chua, S. E. (2007). Stress and psychological distress among SARS survivors 1 year after the outbreak. The Canadian Journal of Psychiatry/La Revue Canadienne de Psychiatrie, 52(4), 233-240. https://doi.org/10.1177/070674370705200405

Li, J., Huang, D. Q., Zou, B., Yang, H., Hui, W. Z., Rui, F., Yee, N., Liu, C., Nerurkar, S. N., Kai, J., Teng, M., Li, X., Zeng, H., Borghi, J. A., Henry, L., Cheung, R., \& Nguyen, M. H. (2021). Epidemiology of COVID-19: A systematic review and meta-analysis of clinical characteristics, risk factors, and outcomes. Journal of Medical Virology, 93(3), 1449-1458. https://doi.org/10.1002/jmv.26424

Mak, I. W. C., Chu, C. M., Pan, P. C., Yiu, M. G. C., \& Chan, V. L. (2009). Long-term psychiatric morbidities among SARS survivors. General Hospital Psychiatry, 31, 318-326. https://doi.org/10.1016/j.genhosppsych.2009.03.001 
Mak, I. W., Chu, C. M., Pan, P. C., Yiu, M. G., Ho, S. C., \& Chan, V. L. (2010). Risk factors for chronic post-traumatic stress disorder (PTSD) in SARS survivors. General Hospital Psychiatry, 32(6), 590-8. https://doi.org/10.1016/j.genhosppsych.2010.07.007

Mazza, M. G., De Lorenzo, R., Conte, C., Poletti, S., Vai, B., Bollettini, I., Melloni, E. M. T., Furlan, R., Ciceri, F., Rovere-Querini, P., Benedetti, F. (2020). Anxiety and depression in COVID-19 survivors: Role of inflammatory and clinical predictors. Brain, Behavior, and Immunity, 89, 594-600. https://doi.org/10.1016/j.bbi.2020.07.037.

McMichael, T. M., Currie, D. W., Clark, S., Pogosjans, S., Kay, M., Schwartz, N. G., Lewis, J., Baer, A., Kawakami, V., Lukoff, M. D., Ferro, J., Brostrom-Smith, C., Rea, T. D., Sayre, M. R., Riedo, F. X., Russell, D., Hiatt, B., Montgomery, P., Rao, A. K.,... \& Duchin, J. S. (2020, Mar 27). Epidemiology of COVID-19 in a long-term care facility in King County, Washington. New England Journal of Medicine, 382(21), 2005-2011. https://doi.org/10.1056/NEJMoa2005412

Parsons, A., Knopp, K., Rhoades, G., Allen, E., Markman, H., \& Stanley, S. (2018). Associations of Army fathers' PTSD symptoms and child functioning: Within- and between-family effects. Family Process, 57(4), 915-926. https://doi.org/10.1111/famp.12358

Prigerson, H. G., Boelen, P. A., Smith, K., Xu, J., \& Maciejewski, P. K. (2021). Validation of newly proposed DSM criteria for prolonged grief disorder and the PG-13-Revised (PG-13-R) scale. World Psychiatry, 20(1), 96-106. https://doi.org/10.1002/wps.20823

Rosenthal, N., Cao, Z., Gundrum, J., Sianis, J., \& Safo, S. (2020). Risk Factors Associated With In-Hospital Mortality in a US National Sample of Patients With COVID-19. JAMA Network Open, 3(12), e2029058. https://doi.org/10.1001/jamanetworkopen.2020.29058

Rytwinski, N. K., Scur, M. D., Feeny, N. C., \& Youngstrom, E. A. (2013). The co-occurrence of major depressive disorder among individuals with posttraumatic stress disorder: A meta-analysis. Journal of traumatic stress, 26(3), 299-309. https://doi.org/10.1002/jts.21814 
Sekowski, M., \& Prigerson, H. G. (2021). Associations between interpersonal dependency and severity of prolonged grief disorder symptoms in bereaved surviving family members. Comprehensive Psychiatry.

Tarsitani, L,. Vassalini, P., Koukopoulos, A., Borrazzo, C., Alessi, F., Di Nicolantonio, C., Serra, R., Alessandri, F., Ceccarelli, G., Mastroianni, C. M., d'Ettorre G. (2021) Posttraumatic stress disorder among COVID-19 survivors at 3-month follow-up after hospital discharge. Journal of General Internal Medicine. Advance online publication. https://doi.org/10.1007/s11606-021-06731-7

The Novel Coronavirus Pneumonia Emergency Response Epidemiology Team. (2020).Vital surveillances: The epidemiological characteristics of an outbreak of 2019 novel coronavirus diseases (COVID-19) - China 2020, February 17, 2020, http://weekly.chinacdc.cn/en/article/id/e53946e2-c6c4-41e9-9a9b-fea8db1a8f51

Thibodeau, M. A., Welch, P. G., Sareen, J., \& Asmundson, G. J. G. (2013). Anxiety disorders are independently associated with suicide ideation and attempts: Propensity score matching in two epidemiological samples. Depression and Anxiety, 30(10), 947-954. https://doi.org/10.1002/da.22203

Vaira, L. A., Deiana, G., Fois, A. G., Pirina, P., Madeddu, G., De Vito, A., Babudieri, S., Petrocelli, M., Serra, A., Bussu, F., Ligas, E., Salzano, G., \& De Riu, G. (2020). Objective evaluation of anosmia and ageusia in COVID-19 patients: Single-center experience on 72 cases. Head \& Neck, 42(6), 1252-1258. https://doi.org/10.1002/hed.26204

World Health Organization. (2020). Report of the WHO-China Joint Mission on Coronavirus Disease 2019 (COVID-19). February 28, 2020. https://www.who.int/docs/defaultsource/coronaviruse/who-china-joint-mission-on-covid-19-final-report.pdf.

World Health Organisation. (2021). Coronavirus (COVID-19) Dashboard, situation by country, territory \& area. April 13, 2021. covid19.who.int 